\title{
Iterative Computation for Solving the Variational Inequality and the Generalized Equilibrium Problem
}

\author{
Xiujuan Pan, ${ }^{1,2}$ Shin Min Kang, ${ }^{3}$ and Young Chel Kwun ${ }^{4}$ \\ ${ }^{1}$ School of Science, Tianjin Polytechnic University, Tianjin 300387, China \\ ${ }^{2}$ College of Management and Economics, Tianjin University, Tianjin 300072, China \\ ${ }^{3}$ Department of Mathematics and the RINS, Gyeongsang National University, Jinju 660-701, Republic of Korea \\ ${ }^{4}$ Department of Mathematics, Dong-A University, Pusan 614-714, Republic of Korea
}

Correspondence should be addressed to Young Chel Kwun; yckwun@dau.ac.kr

Received 22 March 2014; Revised 7 May 2014; Accepted 13 May 2014; Published 27 May 2014

Academic Editor: Giuseppe Marino

Copyright (C) 2014 Xiujuan Pan et al. This is an open access article distributed under the Creative Commons Attribution License, which permits unrestricted use, distribution, and reproduction in any medium, provided the original work is properly cited.

An iterative algorithm for solving the variational inequality and the generalized equilibrium problem has been introduced. Convergence result is given.

\section{Introduction}

Let $\mathbb{Q}$ be a real Hilbert space with inner product $\langle\cdot, \cdot\rangle$ and norm $\|\cdot\|$, respectively. Let $\mathbb{C}$ be a nonempty closed convex subset of $\mathbb{H}$. Recall that a mapping $\mathbb{A}: \mathbb{C} \rightarrow \mathbb{W}$ is said to be

(i) nonexpansive $\Leftrightarrow\|A u-\mathbb{A} v\| \leq\|u-v\|$, for all $u, v \in \mathbb{C}$ (we use $\operatorname{Fix}(\mathbb{A})$ to denote the set of the fixed points of A);

(ii) firmly nonexpansive $\Leftrightarrow\|A u-A v\|^{2} \leq\langle u-v, A u-$ A $v\rangle$, for all $u, v \in \mathbb{C}$;

(iii) $L$-Lipschitz $\Leftrightarrow$ there exists a constant $L>0$ such that $\|A u-A v\| \leq L\|u-v\|$, for all $u, v \in \mathbb{C} ;$

(iv) monotone $\Leftrightarrow\langle u-v, \mathbb{A} u-\mathbb{A} v\rangle \geq 0$, for all $u, v \in \mathbb{C}$;

(v) strongly monotone $\Leftrightarrow$ there exists a constant $\nu>0$ such that $\langle u-v, \mathbb{A} u-\mathbb{A} v\rangle \geq v\|u-v\|^{2}$, for all $u, v \in \mathbb{C}$;

(vi) inverse strongly monotone $\Leftrightarrow$ there exists $\zeta>0$ such that $\langle u-v, \mathbb{A} u-\mathbb{A} v\rangle \geq \zeta\|\mathbb{A} u-\mathbb{A} v\|^{2}$, for all $u, v \in \mathbb{C}$;

(vii) $\zeta$-inverse strongly $\phi$-monotone $\Leftrightarrow\langle\phi(u)-\phi(v), \mathbb{A} u-$ $\mathbb{A} v\rangle \geq \zeta\|A u-A v\|^{2}$, for all $u, v \in \mathbb{C}$ and for some $\zeta>0$, where $\phi: \mathbb{C} \rightarrow \mathbb{C}$ is a nonlinear mapping.

If $\mathbb{A}$ is a multivalued mapping of $H$ into $2^{\natural}$, then $\mathbb{A}$ is said to be a monotone operator on $\mathbb{U} \Leftrightarrow\langle x-y, u-v\rangle \geq 0$, for all $x, y \in \operatorname{dom}(\mathbb{A}), u \in \mathbb{A} x$, and $v \in \mathbb{A} y$. A monotone operator $\mathbb{A}$ on $\mathbb{W}$ is said to be maximal if and only if its graph is not strictly contained in the graph of any other monotone operator on $\mathbb{t}$.

Let $\mathbb{A}, \mathbb{B}$, and $\phi$ be three nonlinear mappings on $\mathbb{C}$. Let $\theta: \mathbb{C} \times \mathbb{C} \rightarrow \mathbb{R}$ be a bifunction. Recall that the equilibrium problem is to find $x^{\dagger} \in \mathbb{C}$ such that

$$
\theta\left(x^{\dagger}, y\right)+\left\langle A x^{\dagger}, y-x^{\dagger}\right\rangle \geq 0, \quad \forall y \in \mathbb{C} .
$$

The solution set of (1) is denoted by $\operatorname{EP}(\theta, \mathbb{A})$. Now we know that the equilibrium theory provides us a natural, novel, and unified framework to study a wide class of problems arising in economics, finance, transportation, network and structural analysis, elasticity, and optimization. For related works, please refer to $[1-3]$ and the references therein.

Recall also that the variational inequality problem is to find $u \in \mathbb{C}, \phi(u) \in \mathbb{C}$ such that

$$
\langle\mathbb{B} u, \phi(v)-\phi(u)\rangle \geq 0, \quad \forall \phi(v) \in \mathbb{C} .
$$

The solution set of $(2)$ is denoted by $\operatorname{VI}(\mathbb{B}, \phi, \mathbb{C})$. It is well known that variational inequality theory has emerged as an important tool in studying a wide class of obstacle, unilateral, free, moving, and equilibrium problems arising in several branches of pure and applied sciences in a unified and general framework. Several numerical methods have been developed for solving variational inequalities and related optimization problems. For related works, please see [4-14]. Noor [15] 
introduced an iterative scheme and studied the approximate solutions of variational inclusion in Hilbert spaces. Glowinski and Le Tallec [16] used the iterative schemes to find the approximate solutions of the elastoviscoplasticity problem, liquid crystal theory, and eigenvalue computation. In 1998, Haubruge et al. [17] studied the convergence analysis of the iterative schemes of Glowinski and Le Tallec and applied these schemes to obtain new splitting-type algorithms for solving variational inequalities, separable convex programming, and minimization of a sum of convex functions.

Our main purpose in the present paper is to solve the following equilibrium problem and variational inequality problem: finding a point $x^{\dagger}$ such that

$$
x^{\dagger} \in V I(\mathbb{B}, \phi, \mathbb{C}), \quad \phi\left(x^{\dagger}\right) \in \operatorname{EP}(\theta, \mathbb{A}) .
$$

Our main motivations are inspired by the following two reasons.

Firstly, it is still an interesting topic for solving the variational inequality problem and the equilibrium problem based on their applications in science and engineering. Secondly, the split problem of finding a point $\tilde{x}$ such that

$$
x^{\dagger} \in \mathbb{C}, \quad \tilde{\phi}\left(x^{\dagger}\right) \in \mathbb{D}
$$

has received much attention. For related works, please refer to [18-20]. However, we observe that the involved operator $\tilde{\phi}$ in (4) is a bounded liner operator. In this paper, we devote to study the problem (3), where the transformation $\phi$ is a nonlinear mapping. For this purpose, we introduce a new iterative algorithm. Consequently, strong convergence analysis is demonstrated.

\section{Preliminaries}

In this section, we recall some useful lemmas.

Recall that the metric projection proj $_{\mathbb{C}}: \mathbb{U} \rightarrow \mathbb{C}$ satisfies $\left\|u-\operatorname{proj}_{\mathbb{C}} u\right\|=\inf \{\|u-v\|: \quad v \in \mathbb{C}\}$. The metric projection $\operatorname{proj}_{\mathbb{C}}$ is a typical firmly nonexpansive mapping. The characteristic inequality of the projection is $\left\langle u-\operatorname{proj}_{\mathbb{C}} u, v-\operatorname{proj}_{\mathbb{C}} u\right\rangle \leq 0$, for all $u \in \mathbb{U}, v \in \mathbb{C}$.

Assume that $\theta: \mathbb{C} \times \mathbb{C} \rightarrow \mathbb{R}$ is a bifunction which satisfies the following conditions:

(C1) $\theta(u, u)=0$, for all $u \in \mathbb{C}$;

(C2) $\theta$ is monotone; that is, $\theta(u, v)+\theta(v, u) \leq 0$, for all $u, v \in \mathbb{C}$;

(C3) for each $u, v, w \in \mathbb{C}, \lim _{t \downarrow 0} \theta(t w+(1-t) u, v) \leq \theta(u, v)$;

(C4) for each $u \in \mathbb{C}, v \mapsto \theta(u, v)$ is convex and lower semicontinuous.

Lemma 1 (see [2]). Let $\mathbb{C}$ be a nonempty closed convex subset of a real Hilbert space $\mathbb{H}$. Let $\theta: \mathbb{C} \times \mathbb{C} \rightarrow \mathbb{R}$ be a bifunction which satisfies conditions (C1)-(C4). Let $\tau>0$ and $u \in \mathbb{C}$. Then, there exists $w \in \mathbb{C}$ such that

$$
\theta(w, v)+\frac{1}{\tau}\langle v-w, w-u\rangle \geq 0, \quad \forall v \in \mathbb{C} .
$$

Further, if $\mathbb{S}_{\tau}(u)=\{w \in \mathbb{C}: \theta(w, v)+(1 / \tau)\langle v-w, w-u\rangle \geq 0$, for all $v \in \mathbb{C}\}$, then the following hold: (a) $\mathbb{S}_{\tau}$ is single-valued and $\mathbb{S}_{\tau}$ is firmly nonexpansive;

(b) $\operatorname{EP}(\theta)$ is closed and convex and $\operatorname{EP}(\theta)=\operatorname{Fix}\left(\mathbb{S}_{\tau}\right)$.

Lemma 2 (see [21]). Let $\left\{x_{n}\right\}$ and $\left\{y_{n}\right\}$ be bounded sequences in a Banach space $\mathbb{X}$ and let $\left\{\eta_{n}\right\}$ be a sequence in $[0,1]$ with $0<\liminf _{n \rightarrow \infty} \eta_{n} \leq \limsup _{n \rightarrow \infty} \eta_{n}<1$. Suppose that $x_{n+1}=$ $\left(1-\eta_{n}\right) y_{n}+\eta_{n} x_{n}$, for all $n \geq 0$, and $\limsup _{n \rightarrow \infty}\left(\left\|y_{n+1}-y_{n}\right\|-\right.$ $\left.\left\|x_{n+1}-x_{n}\right\|\right) \leq 0$. Then, $\lim _{n \rightarrow \infty}\left\|y_{n}-x_{n}\right\|=0$.

Lemma 3 (see [22]). Assume that the sequence $\left\{a_{n}\right\}$ satisfies $a_{n} \geq 0$ and $a_{n+1} \leq\left(1-v_{n}\right) a_{n}+\varsigma_{n} \nu_{n}$, where $\left\{v_{n}\right\}$ is a sequence in $(0,1)$ and $\left\{s_{n}\right\}$ is a sequence such that $\sum_{n=1}^{\infty} \nu_{n}=\infty$ and $\lim \sup _{n \rightarrow \infty} \varsigma_{n} \leq 0\left(\right.$ or $\left.\sum_{n=1}^{\infty}\left|\varsigma_{n} \nu_{n}\right|<\infty\right)$. Then, $\lim _{n \rightarrow \infty} a_{n}=$ 0 .

\section{Main Results}

Let $\mathbb{C}$ be a nonempty closed convex subset of a real Hilbert space $\mathbb{U}$. Let $\mathbb{A}: \mathbb{C} \rightarrow \mathbb{U}$ be an $\eta$-inverse strongly monotone mapping. Let $\phi: \mathbb{C} \rightarrow \mathbb{C}$ be a weakly continuous and $\nu$ strongly monotone mapping such that $R(\phi)=\mathbb{C}$. Let $\mathbb{B}$ : $\mathbb{C} \rightarrow \mathbb{H}$ be a $\zeta$-inverse strongly $\phi$-monotone mapping. Let $\varrho: \mathbb{C} \rightarrow \mathbb{W}$ be an L-Lipschitz continuous mapping. Let $\theta:$ $\mathbb{C} \times \mathbb{C} \rightarrow \mathbb{R}$ be a bifunction which satisfies $(\mathrm{C} 1)-(\mathrm{C} 4)$ in the above section. Let $\left\{\zeta_{n}\right\} \subset[0,1],\left\{\eta_{n}\right\} \subset[0,1],\left\{\omega_{n}\right\} \subset(0, \infty)$, and $\left\{\tau_{n}\right\} \subset(0, \infty)$ be four real number sequences and let $\varsigma>0$ be a constant.

We use $\Delta$ to denote the solution set of (3). In order to solve (3), we introduce the following three-step algorithm.

Algorithm 4. Let $x_{0} \in \mathbb{C}$ be an initial guess. Define the sequence $\left\{x_{n}\right\}$ as follows:

$$
\begin{gathered}
u_{n}=\operatorname{proj}_{\mathbb{C}}\left[\phi\left(x_{n}\right)-\omega_{n} \mathbb{B} x_{n}\right], \quad n \geq 0, \\
\theta\left(z_{n}, y\right)+\left\langle A u_{n}, y-z_{n}\right\rangle+\frac{1}{\tau_{n}}\left\langle y-z_{n}, z_{n}-u_{n}\right\rangle \geq 0, \\
\forall y \in \mathbb{C}, \\
\phi\left(x_{n+1}\right) \quad n \geq 0 . \\
=\eta_{n} \phi\left(x_{n}\right)+\left(1-\eta_{n}\right) \operatorname{proj}_{\mathbb{C}}\left[\zeta_{n} \varsigma Q\left(x_{n}\right)+\left(1-\zeta_{n}\right) z_{n}\right], \\
n \geq 0
\end{gathered}
$$

Theorem 5. Suppose that $\Delta \neq \emptyset$. Assume that the following conditions are satisfied:

(r1) $\Phi_{n} \in\left(a_{1}, a_{2}\right) \subset(0,2 \zeta)$ and $\lim _{n \rightarrow \infty}\left(\omega_{n+1}-\omega_{n}\right)=0$;

(r2) $\tau_{n} \in\left(a_{3}, a_{4}\right) \subset(0,2 \eta)$ and $\lim _{n \rightarrow \infty}\left(\tau_{n+1}-\tau_{n}\right)=0$;

(r3) $\eta_{n} \in\left[a_{5}, a_{6}\right] \subset(0,1)$;

(r4) $\lim _{n \rightarrow \infty} \zeta_{n}=0$ and $\sum_{n} \zeta_{n}=\infty$;

(r5) $v \in(L \varsigma, 2 \zeta)$

Then, the sequence $\left\{x_{n}\right\}$ generated by (6) converges strongly to $x^{*} \in \Delta$ which solves the following variational inequality:

$$
\left\langle\varsigma \varrho\left(x^{*}\right)-\phi\left(x^{*}\right), \phi(x)-\phi\left(x^{*}\right)\right\rangle \leq 0, \quad \forall x \in \Delta .
$$


Proof. First of all, we prove that the solution of the variational inequality (7) is unique. In fact, if $\tilde{x} \in \Delta$ also solves (7), then we get

$$
\begin{gathered}
\left\langle\varsigma \varrho\left(x^{*}\right)-\phi\left(x^{*}\right), \phi(\tilde{x})-\phi\left(x^{*}\right)\right\rangle \leq 0 \\
\left\langle\varsigma \varrho(\tilde{x})-\phi(\tilde{x}), \phi\left(x^{*}\right)-\phi(\tilde{x})\right\rangle \leq 0
\end{gathered}
$$

It follows that

$$
\left\langle\varsigma \varrho(\tilde{x})-\phi(\tilde{x})-\varsigma \varrho\left(x^{*}\right)+\phi\left(x^{*}\right), \phi\left(x^{*}\right)-\phi(\tilde{x})\right\rangle \leq 0 .
$$

So,

$$
\begin{aligned}
\left\|\phi\left(x^{*}\right)-\phi(\tilde{x})\right\|^{2} & \leq \varsigma\left\langle\varrho\left(x^{*}\right)-\varrho(\tilde{x}), \phi\left(x^{*}\right)-\phi(\tilde{x})\right\rangle \\
& \leq \varsigma\left\|\varrho\left(x^{*}\right)-\varrho(\tilde{x})\right\|\left\|\phi\left(x^{*}\right)-\phi(\tilde{x})\right\|,
\end{aligned}
$$

which implies that

$$
\left\|\phi\left(x^{*}\right)-\phi(\tilde{x})\right\| \leq \varsigma\left\|\varrho\left(x^{*}\right)-\varrho(\tilde{x})\right\| .
$$

Since $\phi$ is $\nu$-strongly monotone, we have

$$
\begin{aligned}
v\left\|x^{*}-\tilde{x}\right\|^{2} & \leq\left\langle\phi\left(x^{*}\right)-\phi(\tilde{x}), x^{*}-\tilde{x}\right\rangle \\
& \leq\left\|\phi\left(x^{*}\right)-\phi(\tilde{x})\right\|\left\|x^{*}-\tilde{x}\right\| .
\end{aligned}
$$

Thus,

$$
\begin{aligned}
\nu\left\|x^{*}-\tilde{x}\right\| & \leq\left\|\phi\left(x^{*}\right)-\phi(\tilde{x})\right\| \\
& \leq \varsigma\left\|\varrho\left(x^{*}\right)-\varrho(\tilde{x})\right\| \leq \varsigma L\left\|x^{*}-\tilde{x}\right\| .
\end{aligned}
$$

Since $\varsigma L<v$, we deduce the contradiction. Therefore, $x^{*}=\tilde{x}$. So, the solution of variational inequality (7) is unique.

Let $x^{\#} \in \Delta$. Hence, $x^{\#} \in V I(\mathbb{B}, \phi, \mathbb{C})$ and $\phi\left(x^{\#}\right) \in$ $\operatorname{EP}(\theta, \mathbb{A})$. Note that

$$
\begin{array}{r}
x^{\#} \in V I(\mathbb{B}, \phi, \mathbb{C}) \Longleftrightarrow \phi\left(x^{\#}\right)=\operatorname{proj}_{\mathbb{C}}\left(\phi\left(x^{\#}\right)-v \mathbb{B} x^{\#}\right), \\
\forall v>0 .
\end{array}
$$

Since $\omega_{n}>0$, we have $\phi\left(x^{\#}\right)=\operatorname{proj}_{\mathbb{C}}\left[\phi\left(x^{\#}\right)-\omega_{n} \mathbb{B} x^{\#}\right]$, for all $n \geq 0$. For $u, v \in \mathbb{C}$, we have

$$
\begin{aligned}
\left\|\left(\phi(u)-\omega_{n} \mathbb{B} u\right)-\left(\phi(v)-\omega_{n} \mathbb{B} v\right)\right\|^{2} \\
=\|\phi(u)-\phi(v)\|^{2}-2 \omega_{n}\langle\mathbb{B} u-\mathbb{B} v, \phi(u)-\phi(v)\rangle \\
\quad+\omega_{n}^{2}\|\mathbb{B} u-\mathbb{B} v\|^{2} \\
\leq\|\phi(u)-\phi(v)\|^{2}-2 \omega_{n} \zeta\|\mathbb{B} u-\mathbb{B} v\|^{2}+\omega_{n}^{2}\|\mathbb{B} u-\mathbb{B} v\|^{2} \\
\leq\|\phi(u)-\phi(v)\|^{2}+\omega_{n}\left(\omega_{n}-2 \zeta\right)\|\mathbb{B} u-\mathbb{B} v\|^{2} .
\end{aligned}
$$

Hence,

$$
\left\|\left(\phi(u)-\omega_{n} \mathbb{B} u\right)-\left(\phi(v)-\omega_{n} \mathbb{B} v\right)\right\| \leq\|\phi(u)-\phi(v)\|,
$$

for all $u, v \in \mathbb{C}$. Thus,

$$
\left\|u_{n}-\phi\left(x^{\#}\right)\right\|
$$$$
=\left\|\operatorname{proj}_{\mathbb{C}}\left[\phi\left(x_{n}\right)-\omega_{n} \mathbb{B} x_{n}\right]-\operatorname{proj}_{\mathbb{C}}\left[\phi\left(x^{\#}\right)-\omega_{n} \mathbb{B} x^{\#}\right]\right\|
$$$$
\leq\left\|\left(\phi\left(x_{n}\right)-\omega_{n} \mathbb{B} x_{n}\right)-\left(\phi\left(x^{\#}\right)-\omega_{n} \mathbb{B} x^{\#}\right)\right\|
$$$$
\leq\left\|\phi\left(x_{n}\right)-\phi\left(x^{\#}\right)\right\|
$$

From (6), we have $z_{n}=\mathbb{S}_{\tau_{n}}\left(I-\tau_{n} \mathbb{A}\right) u_{n}$, for all $n \geq 0$. Noting that $\phi\left(x^{\#}\right) \in E P(\theta, \mathbb{A})$, we deduce $\phi\left(x^{\#}\right)=\mathbb{S}_{\tau_{n}}\left(I-\tau_{n} \mathbb{A}\right) \phi\left(x^{\#}\right)$, for all $n \geq 0$. It follows from (17) that

$$
\begin{aligned}
\| & \phi\left(x_{n+1}\right)-\phi\left(x^{\#}\right) \| \\
\leq & \eta_{n}\left\|\phi\left(x_{n}\right)-\phi\left(x^{\#}\right)\right\| \\
& +\left(1-\eta_{n}\right)\left\|\operatorname{proj}_{\mathbb{C}}\left[\zeta_{n} \varsigma \varrho\left(x_{n}\right)+\left(1-\zeta_{n}\right) z_{n}\right]-\phi\left(x^{\#}\right)\right\| \\
\leq & \eta_{n}\left\|\phi\left(x_{n}\right)-\phi\left(x^{\#}\right)\right\| \\
& +\left(1-\eta_{n}\right)\left\|\zeta_{n}\left(\varsigma \varrho\left(x_{n}\right)-\phi\left(x^{\#}\right)\right)+\left(1-\zeta_{n}\right)\left(z_{n}-\phi\left(x^{\#}\right)\right)\right\| \\
\leq & \eta_{n}\left\|\phi\left(x_{n}\right)-\phi\left(x^{\#}\right)\right\| \\
& +\left(1-\eta_{n}\right)\left[\zeta_{n}\left\|\varsigma \varrho\left(x_{n}\right)-\varsigma \varrho\left(x^{\#}\right)\right\|+\zeta_{n}\left\|\varsigma \varrho\left(x^{\#}\right)-\phi\left(x^{\#}\right)\right\|\right. \\
& +\left(1-\zeta_{n}\right) \\
& +\left(1-\eta_{n}\right)\left[\frac{\zeta_{n} \varsigma L}{v}\left\|\phi\left(x_{n}\right)-\phi\left(x^{\#}\right)\right\|+\zeta_{n}\left\|\varsigma \varrho\left(x^{\#}\right)-\phi\left(x^{\#}\right)\right\|\right. \\
& +\left(1-\eta_{n}\left(I-\tau_{n} \mathbb{A}\right) u_{n}-\mathbb{S}_{\tau_{n}}\left(I-\tau_{n} \mathbb{A}\right) \phi\left(x^{\#}\right) \|\right] \\
& +\eta_{n}\left\|\phi\left(x_{n}\right)-\phi\left(x^{\#}\right)\right\| \\
& +\left(1-\eta_{n}\right)\left[\zeta_{n} \varsigma L\left\|x_{n}-x^{\#}\right\|+\zeta_{n}\left\|\varsigma \varrho\left(x^{\#}\right)-\phi\left(x^{\#}\right)\right\|\right. \\
& +\phi\left(x^{\#}\right) \|
\end{aligned}
$$


An induction implies that

$$
\begin{aligned}
& \left\|\phi\left(x_{n}\right)-\phi\left(x^{\#}\right)\right\| \\
& \quad \leq \max \left\{\left\|\phi\left(x_{0}\right)-\phi\left(x^{\#}\right)\right\|, \frac{\left\|\varsigma \varrho\left(x^{\#}\right)-\phi\left(x^{\#}\right)\right\|}{1-\varsigma L / \nu}\right\} .
\end{aligned}
$$

Hence, $\left\{\phi\left(x_{n}\right)\right\}$ is bounded. Since $\phi$ is $\nu$-strongly monotone, we can deduce $\nu\left\|x_{n}-x^{\#}\right\| \leq\left\|\phi\left(x_{n}\right)-\phi\left(x^{\#}\right)\right\|$. So,

$$
\begin{aligned}
\| x_{n}- & x^{\#} \| \\
& \leq \frac{1}{v}\left\|\phi\left(x_{n}\right)-\phi\left(x^{\#}\right)\right\| \\
& \leq \frac{1}{v} \max \left\{\left\|\phi\left(x_{0}\right)-\phi\left(x^{\#}\right)\right\|, \frac{\left\|\varsigma \varrho\left(x^{\#}\right)-\phi\left(x^{\#}\right)\right\|}{1-\varsigma L / \nu}\right\} .
\end{aligned}
$$

This implies that $\left\{x_{n}\right\}$ is bounded.

From (6), we have

$$
\theta\left(z_{n}, y\right)+\frac{1}{\tau_{n}}\left\langle y-z_{n}, z_{n}-\left(u_{n}-\tau_{n} \mathbb{A} u_{n}\right)\right\rangle \geq 0, \quad \forall y \in \mathbb{C} .
$$

So,

$$
\theta\left(z_{n}, z_{n+1}\right)+\frac{1}{\tau_{n}}\left\langle z_{n+1}-z_{n}, z_{n}-\left(u_{n}-\tau_{n} A u_{n}\right)\right\rangle \geq 0 .
$$

Similarly,

$$
\begin{aligned}
& \theta\left(z_{n+1}, z_{n}\right)+\frac{1}{\tau_{n+1}}\left\langle z_{n}-z_{n+1}, z_{n+1}-\left(u_{n+1}-\tau_{n+1} \mathbb{A} u_{n+1}\right)\right\rangle \\
& \quad \geq 0 .
\end{aligned}
$$

Hence,

$$
\begin{gathered}
\theta\left(z_{n}, z_{n+1}\right)+\theta\left(z_{n+1}, z_{n}\right)+\left\langle A u_{n}-\mathrm{A} u_{n+1}, z_{n+1}-z_{n}\right\rangle \\
+\left\langle z_{n+1}-z_{n}, \frac{z_{n}-u_{n}}{\tau_{n}}-\frac{z_{n+1}-u_{n+1}}{\tau_{n+1}}\right\rangle \geq 0
\end{gathered}
$$

Since $\theta$ is monotone, we have

$$
\theta\left(z_{n}, z_{n+1}\right)+\theta\left(z_{n+1}, z_{n}\right) \leq 0 .
$$

So,

$$
\begin{aligned}
& \left\langle\mathcal{A} u_{n}-\mathbb{A} u_{n+1}, z_{n+1}-z_{n}\right\rangle \\
& \quad+\left\langle z_{n+1}-z_{n}, \frac{z_{n}-u_{n}}{\tau_{n}}-\frac{z_{n+1}-u_{n+1}}{\tau_{n+1}}\right\rangle \geq 0 .
\end{aligned}
$$

Thus,

$$
\begin{aligned}
& \tau_{n}\left\langle\mathrm{~A} u_{n}-\mathrm{A} u_{n+1}, z_{n+1}-z_{n}\right\rangle \\
& \quad+\left\langle z_{n+1}-z_{n}, z_{n}-z_{n+1}+z_{n+1}-u_{n}-\frac{\tau_{n}}{\tau_{n+1}}\left(z_{n+1}-u_{n+1}\right)\right\rangle
\end{aligned}
$$$$
\geq 0 \text {. }
$$

It follows that

$$
\begin{aligned}
\| z_{n+1} & -z_{n} \|^{2} \\
\leq & \tau_{n}\left\langle\mathbb{A} u_{n}-\mathbb{A} u_{n+1}, z_{n+1}-z_{n}\right\rangle \\
& +\left\langle z_{n+1}-z_{n}, u_{n+1}-u_{n}+\left(1-\frac{\tau_{n}}{\tau_{n+1}}\right)\left(z_{n+1}-u_{n+1}\right)\right\rangle \\
= & \left\langle\left(I-\tau_{n} \mathbb{A}\right) u_{n+1}-\left(I-\tau_{n} \mathbb{A}\right) u_{n}, z_{n+1}-z_{n}\right\rangle \\
& +\left\langle z_{n+1}-z_{n},\left(1-\frac{\tau_{n}}{\tau_{n+1}}\right)\left(z_{n+1}-u_{n+1}\right)\right\rangle \\
\leq & \left\|\left(I-\tau_{n} \mathbb{A}\right) u_{n+1}-\left(I-\tau_{n} \mathbb{A}\right) u_{n}\right\|\left\|z_{n+1}-z_{n}\right\| \\
& +\left|1-\frac{\tau_{n}}{\tau_{n+1}}\right|\left\|z_{n+1}-z_{n}\right\|\left\|z_{n+1}-u_{n+1}\right\| \\
\leq & \left\|z_{n+1}-z_{n}\right\|\left(\left\|u_{n+1}-u_{n}\right\|+\left|1-\frac{\tau_{n}}{\tau_{n+1}}\right|\left\|z_{n+1}-u_{n+1}\right\|\right)
\end{aligned}
$$

and hence

$$
\begin{aligned}
\left\|z_{n+1}-z_{n}\right\| & \leq\left\|u_{n+1}-u_{n}\right\|+\left|1-\frac{\tau_{n}}{\tau_{n+1}}\right|\left\|z_{n+1}-u_{n+1}\right\| \\
& \leq\left\|u_{n+1}-u_{n}\right\|+\frac{1}{a_{3}}\left|\tau_{n+1}-\tau_{n}\right|\left\|z_{n+1}-u_{n+1}\right\| .
\end{aligned}
$$

By (6) and (16), we have

$$
\begin{aligned}
& \left\|u_{n+1}-u_{n}\right\| \\
= & \left\|\operatorname{proj}_{\mathbb{C}}\left[\phi\left(x_{n+1}\right)-\omega_{n+1} \mathbb{B} x_{n+1}\right]-\operatorname{proj}_{\mathbb{C}}\left[\phi\left(x_{n}\right)-\omega_{n} \mathbb{B} x_{n}\right]\right\| \\
\leq & \left\|\left[\phi\left(x_{n+1}\right)-\omega_{n+1} \mathbb{B} x_{n+1}\right]-\left[\phi\left(x_{n}\right)-\omega_{n} \mathbb{B} x_{n}\right]\right\| \\
\leq & \left\|\phi\left(x_{n+1}\right)-\omega_{n+1} \mathbb{B} x_{n+1}-\left(\phi\left(x_{n}\right)-\omega_{n+1} \mathbb{B} x_{n}\right)\right\| \\
& +\left|\omega_{n+1}-\omega_{n}\right|\left\|\mathbb{B}\left(x_{n}\right)\right\| \\
\leq & \left\|\phi\left(x_{n+1}\right)-\phi\left(x_{n}\right)\right\|+\left|\omega_{n+1}-\omega_{n}\right|\left\|\mathbb{B}\left(x_{n}\right)\right\| .
\end{aligned}
$$

Therefore,

$$
\begin{aligned}
& \left\|z_{n+1}-z_{n}\right\| \\
& \quad \leq\left\|\phi\left(x_{n+1}\right)-\phi\left(x_{n}\right)\right\| \\
& \quad+\left|\omega_{n+1}-\omega_{n}\right|\left\|\mathbb{B}\left(x_{n}\right)\right\|+\frac{1}{a_{3}}\left|\tau_{n+1}-\tau_{n}\right|\left\|z_{n+1}-u_{n+1}\right\| .
\end{aligned}
$$

It follows that

$$
\begin{aligned}
& \left\|z_{n+1}-z_{n}\right\|-\left\|\phi\left(x_{n+1}\right)-\phi\left(x_{n}\right)\right\| \\
& \quad \leq\left|\omega_{n+1}-\omega_{n}\right|\left\|\mathbb{B}\left(x_{n}\right)\right\|+\frac{1}{a_{3}}\left|\tau_{n+1}-\tau_{n}\right|\left\|z_{n+1}-u_{n+1}\right\| .
\end{aligned}
$$


Since $\lim _{n \rightarrow \infty}\left(\Phi_{n+1}-\Phi_{n}\right)=0, \lim _{n \rightarrow \infty}\left(\tau_{n+1}-\tau_{n}\right)=0$, and the sequences $\left\{\varrho\left(x_{n}\right)\right\},\left\{\phi\left(x_{n}\right)\right\},\left\{z_{n}\right\},\left\{u_{n}\right\}$, and $\left\{\mathbb{B} x_{n}\right\}$ are bounded, we have

$$
\limsup _{n \rightarrow \infty}\left(\left\|z_{n+1}-z_{n}\right\|-\left\|\phi\left(x_{n+1}\right)-\phi\left(x_{n}\right)\right\|\right) \leq 0 .
$$

From Lemma 2, we obtain

$$
\lim _{n \rightarrow \infty}\left\|z_{n}-\phi\left(x_{n}\right)\right\|=0 .
$$

Note that

$$
\begin{aligned}
\left\|\phi\left(x_{n+1}\right)-\phi\left(x_{n}\right)\right\| \leq & \left(1-\eta_{n}\right) \zeta_{n}\left\|\varsigma \varrho\left(x_{n}\right)-\phi\left(x_{n}\right)\right\| \\
& +\left(1-\eta_{n}\right)\left(1-\zeta_{n}\right)\left\|z_{n}-\phi\left(x_{n}\right)\right\| .
\end{aligned}
$$

Hence,

$$
\lim _{n \rightarrow \infty}\left\|\phi\left(x_{n+1}\right)-\phi\left(x_{n}\right)\right\|=0 .
$$

This together with the $v$-strong monotonicity of $\phi$ implies that

$$
\lim _{n \rightarrow \infty}\left\|x_{n+1}-x_{n}\right\|=0
$$

From (18), we have

$$
\begin{aligned}
& \left\|\phi\left(x_{n+1}\right)-\phi\left(x^{\#}\right)\right\| \\
& \leq \eta_{n}\left\|\phi\left(x_{n}\right)-\phi\left(x^{\#}\right)\right\| \\
& +\left(1-\eta_{n}\right)\left[\zeta_{n} \varsigma L\left\|x_{n}-x^{\#}\right\|+\zeta_{n}\left\|\varsigma \varrho\left(x^{\#}\right)-\phi\left(x^{\#}\right)\right\|\right. \\
& \left.+\left(1-\zeta_{n}\right)\left\|u_{n}-\phi\left(x^{\#}\right)\right\|\right] \\
& \leq \eta_{n}\left\|\phi\left(x_{n}\right)-\phi\left(x^{\#}\right)\right\| \\
& +\left(1-\eta_{n}\right)\left[\frac{\zeta_{n} \varsigma L}{v}\left\|\phi\left(x_{n}\right)-\phi\left(x^{\#}\right)\right\|\right. \\
& \quad+\zeta_{n}\left\|\varsigma \varrho\left(x^{\#}\right)-\phi\left(x^{\#}\right)\right\| \\
& \left.\quad+\left(1-\zeta_{n}\right)\left\|u_{n}-\phi\left(x^{\#}\right)\right\|\right] \\
& +\left(1-\left(1-\eta_{n}\right)\left(1-\frac{\varsigma L \zeta_{n}}{v}\right)\right]\left\|\phi\left(x_{n}\right)-\phi\left(x^{\#}\right)\right\| \\
& +\left(1-\eta_{n}\right)\left(1-\eta_{n}\right) \| u_{n}-\phi\left(1-\frac{\varsigma L}{v}\right) \frac{\left\|\varsigma \varrho\left(x^{\#}\right)\right\|}{1-\varsigma L / v}
\end{aligned}
$$

By the convexity of the norm and (17), we have

$$
\begin{aligned}
& \left\|\phi\left(x_{n+1}\right)-\phi\left(x^{\#}\right)\right\|^{2} \\
& \leq\left[1-\left(1-\eta_{n}\right)\left(1-\frac{\varsigma L \zeta_{n}}{v}\right)\right]\left\|\phi\left(x_{n}\right)-\phi\left(x^{\#}\right)\right\|^{2} \\
& +\left(1-\zeta_{n}\right)\left(1-\eta_{n}\right)\left\|u_{n}-\phi\left(x^{\#}\right)\right\|^{2} \\
& +\left(1-\eta_{n}\right) \zeta_{n}\left(1-\frac{\varsigma L}{\nu}\right)\left(\frac{\left\|\varsigma \varrho\left(x^{\#}\right)-\phi\left(x^{\#}\right)\right\|}{1-\varsigma L / \nu}\right)^{2} \\
& \leq\left[1-\left(1-\eta_{n}\right)\left(1-\frac{\varsigma L \zeta_{n}}{v}\right)\right]\left\|\phi\left(x_{n}\right)-\phi\left(x^{\#}\right)\right\|^{2} \\
& +\left(1-\zeta_{n}\right)\left(1-\eta_{n}\right) \\
& \times\left\|\left(\phi\left(x_{n}\right)-\omega_{n} \mathbb{B} x_{n}\right)-\left(\phi\left(x^{\#}\right)-\varrho_{n} \mathbb{B} x^{\#}\right)\right\|^{2} \\
& +\left(1-\eta_{n}\right) \zeta_{n}\left(1-\frac{\varsigma L}{\nu}\right)\left(\frac{\left\|\varsigma \varrho\left(x^{\#}\right)-\phi\left(x^{\#}\right)\right\|}{1-\varsigma L / \nu}\right)^{2} \\
& \leq\left[1-\left(1-\eta_{n}\right)\left(1-\frac{\varsigma L \zeta_{n}}{\nu}\right)\right]\left\|\phi\left(x_{n}\right)-\phi\left(x^{\#}\right)\right\|^{2} \\
& +\left(1-\zeta_{n}\right)\left(1-\eta_{n}\right) \\
& \times\left(\left\|\phi\left(x_{n}\right)-\phi\left(x^{\#}\right)\right\|^{2}+\Phi_{n}\left(\varrho_{n}-2 \zeta\right)\left\|\mathbb{B} x_{n}-\mathbb{B} x^{\#}\right\|^{2}\right) \\
& +\left(1-\eta_{n}\right) \zeta_{n}\left(1-\frac{\varsigma L}{v}\right)\left(\frac{\left\|\varsigma \varrho\left(x^{\#}\right)-\phi\left(x^{\#}\right)\right\|}{1-\varsigma L / v}\right)^{2} \\
& \leq\left\|\phi\left(x_{n}\right)-\phi\left(x^{\#}\right)\right\|^{2} \\
& +\left(1-\zeta_{n}\right)\left(1-\eta_{n}\right) \omega_{n}\left(\omega_{n}-2 \zeta\right)\left\|\mathbb{B} x_{n}-\mathbb{B} x^{\#}\right\|^{2} \\
& +\left(1-\eta_{n}\right) \zeta_{n}\left(1-\frac{\varsigma L}{\nu}\right)\left(\frac{\left\|\varsigma \varrho\left(x^{\#}\right)-\phi\left(x^{\#}\right)\right\|}{1-\varsigma L / \nu}\right)^{2} .
\end{aligned}
$$

So,

$$
\begin{aligned}
(1- & \left.\eta_{n}\right)\left(1-\zeta_{n}\right) \Phi_{n}\left(2 \zeta-\Phi_{n}\right)\left\|\mathbb{B} x_{n}-\mathbb{B} x^{\#}\right\|^{2} \\
\leq & \left\|\phi\left(x_{n}\right)-\phi\left(x^{\#}\right)\right\|^{2}-\left\|\phi\left(x_{n+1}\right)-\phi\left(x^{\#}\right)\right\|^{2} \\
& +\left(1-\eta_{n}\right) \zeta_{n}\left(1-\frac{\varsigma L}{v}\right)\left(\frac{\left\|\varsigma \varrho\left(x^{\#}\right)-\phi\left(x^{\#}\right)\right\|}{1-\varsigma L / v}\right)^{2} \\
\leq & \left(\left\|\phi\left(x_{n}\right)-\phi\left(x^{\#}\right)\right\|+\left\|\phi\left(x_{n+1}\right)-\phi\left(x^{\#}\right)\right\|\right) \\
& \times\left\|\phi\left(x_{n+1}\right)-\phi\left(x_{n}\right)\right\| \\
& +\left(1-\eta_{n}\right) \zeta_{n}\left(1-\frac{\varsigma L}{v}\right)\left(\frac{\left\|\varsigma \varrho\left(x^{\#}\right)-\phi\left(x^{\#}\right)\right\|}{1-\varsigma L / v}\right)^{2} .
\end{aligned}
$$


Since $\zeta_{n} \rightarrow 0,\left\|\phi\left(x_{n+1}\right)-\phi\left(x_{n}\right)\right\| \rightarrow 0$, and $\liminf _{n \rightarrow \infty}(1-$ $\left.\eta_{n}\right)\left(1-\zeta_{n}\right) \omega_{n}\left(2 \zeta-\omega_{n}\right)>0$, we obtain

$$
\lim _{n \rightarrow \infty}\left\|\mathbb{B} x_{n}-\mathbb{B} x^{\#}\right\|=0 .
$$

Note that

$$
\begin{aligned}
& \left\|u_{n}-\phi\left(x^{\#}\right)\right\|^{2} \\
& =\left\|\operatorname{proj}_{\mathbb{C}}\left[\phi\left(x_{n}\right)-\omega_{n} \mathbb{B} x_{n}\right]-\operatorname{proj}_{\mathbb{C}}\left[\phi\left(x^{\#}\right)-\omega_{n} \mathbb{B} x^{\#}\right]\right\|^{2} \\
& \leq\left\langle\phi\left(x_{n}\right)-\omega_{n} \mathbb{B} x_{n}-\left(\phi\left(x^{\#}\right)-\omega_{n} \mathbb{B} x^{\#}\right), u_{n}-\phi\left(x^{\#}\right)\right\rangle \\
& =\frac{1}{2}\left\{\left\|\phi\left(x_{n}\right)-\omega_{n} \mathbb{B} x_{n}-\left(\phi\left(x^{\#}\right)-\omega_{n} \mathbb{B} x^{\#}\right)\right\|^{2}\right. \\
& \quad+\left\|u_{n}-\phi\left(x^{\#}\right)\right\|^{2} \\
& \left.\quad-\left\|\phi\left(x_{n}\right)-\omega_{n} \mathbb{B} x_{n}-\left(\phi\left(x^{\#}\right)-\omega_{n} \mathbb{B} x^{\#}\right)-u_{n}+\phi\left(x^{\#}\right)\right\|^{2}\right\} \\
& \leq \frac{1}{2}\left\{\left\|\phi\left(x_{n}\right)-\phi\left(x^{\#}\right)\right\|^{2}+\left\|u_{n}-\phi\left(x^{\#}\right)\right\|^{2}\right. \\
& \left.\quad-\left\|\phi\left(x_{n}\right)-u_{n}-\omega_{n}\left(\mathbb{B} x_{n}-\mathbb{B} x^{\#}\right)\right\|^{2}\right\} \\
& \left.\quad+2 \omega_{n}\left\langle\phi\left(x_{n}\right)-u_{n}, \mathbb{B} x_{n}-\mathbb{B} x^{\#}\right\rangle\right\} . \\
& \quad \frac{1}{2}\left\{\left\|\phi\left(x_{n}\right)-\phi\left(x^{\#}\right)\right\|^{2}+\left\|u_{n}-\phi\left(x^{\#}\right)\right\|^{2}\right. \\
&
\end{aligned}
$$

It follows that

$$
\begin{aligned}
\left\|u_{n}-\phi\left(x^{\#}\right)\right\|^{2} & \\
\leq & \left\|\phi\left(x_{n}\right)-\phi\left(x^{\#}\right)\right\|^{2}-\left\|\phi\left(x_{n}\right)-u_{n}\right\|^{2}-\Phi_{n}^{2}\left\|\mathbb{B} x_{n}-\mathbb{B} x^{\#}\right\|^{2} \\
& +2 \omega_{n}\left\|\phi\left(x_{n}\right)-u_{n}\right\|\left\|\mathbb{B} x_{n}-\mathbb{B} x^{\#}\right\| .
\end{aligned}
$$

From (39) and (43), we have

$$
\begin{aligned}
& \left\|\phi\left(x_{n+1}\right)-\phi\left(x^{\#}\right)\right\|^{2} \\
& \leq\left[1-\left(1-\eta_{n}\right)\left(1-\frac{\varsigma L \zeta_{n}}{v}\right)\right]\left\|\phi\left(x_{n}\right)-\phi\left(x^{\#}\right)\right\|^{2} \\
& \quad+\left(1-\zeta_{n}\right)\left(1-\eta_{n}\right)\left\|u_{n}-\phi\left(x^{\#}\right)\right\|^{2} \\
& \quad+\left(1-\eta_{n}\right) \zeta_{n}\left(1-\frac{\varsigma L}{\nu}\right)\left(\frac{\left\|\varsigma \varrho\left(x^{\#}\right)-\phi\left(x^{\#}\right)\right\|}{1-\varsigma L / \nu}\right)^{2}
\end{aligned}
$$

$$
\begin{aligned}
\leq & {\left[1-\left(1-\eta_{n}\right)\left(1-\frac{\varsigma L \zeta_{n}}{v}\right)\right]\left\|\phi\left(x_{n}\right)-\phi\left(x^{\#}\right)\right\|^{2} } \\
& +\left(1-\zeta_{n}\right)\left(1-\eta_{n}\right)\left\|\phi\left(x_{n}\right)-\phi\left(x^{\#}\right)\right\|^{2} \\
& -\left(1-\zeta_{n}\right)\left(1-\eta_{n}\right)\left\|\phi\left(x_{n}\right)-u_{n}\right\|^{2} \\
& +2\left(1-\zeta_{n}\right)\left(1-\eta_{n}\right) \omega_{n}\left\|\phi\left(x_{n}\right)-u_{n}\right\|\left\|\mathbb{B} x_{n}-\mathbb{B} x^{\#}\right\| \\
& +\left(1-\eta_{n}\right) \zeta_{n}\left(1-\frac{\varsigma L}{v}\right)\left(\frac{\left\|\varsigma \varrho\left(x^{\#}\right)-\phi\left(x^{\#}\right)\right\|}{1-\varsigma L / v}\right)^{2} \\
\leq & \left\|\phi\left(x_{n}\right)-\phi\left(x^{\#}\right)\right\|^{2}-\left(1-\zeta_{n}\right)\left(1-\eta_{n}\right)\left\|\phi\left(x_{n}\right)-u_{n}\right\|^{2} \\
& +2 \omega_{n}\left\|\phi\left(x_{n}\right)-u_{n}\right\|\left\|\mathbb{B} x_{n}-\mathbb{B} x^{\#}\right\| \\
& +\left(1-\eta_{n}\right) \zeta_{n}\left(1-\frac{\varsigma L}{v}\right)\left(\frac{\left\|\varsigma \varrho\left(x^{\#}\right)-\phi\left(x^{\#}\right)\right\|}{1-\varsigma L / v}\right)^{2} .
\end{aligned}
$$

Then, we obtain

$$
\begin{aligned}
&\left(1-\zeta_{n}\right)\left(1-\eta_{n}\right)\left\|\phi\left(x_{n}\right)-u_{n}\right\|^{2} \\
& \leq\left(\left\|\phi\left(x_{n}\right)-\phi\left(x^{\#}\right)\right\|+\left\|\phi\left(x_{n+1}\right)-\phi\left(x^{\#}\right)\right\|\right) \\
& \quad \times\left\|\phi\left(x_{n+1}\right)-\phi\left(x_{n}\right)\right\| \\
&+2 \omega_{n}\left\|\phi\left(x_{n}\right)-u_{n}\right\|\left\|\mathbb{B} x_{n}-\mathbb{B} x^{\#}\right\| \\
&+\left(1-\eta_{n}\right) \zeta_{n}\left(1-\frac{\varsigma L}{v}\right)\left(\frac{\left\|\varsigma \varrho\left(x^{\#}\right)-\phi\left(x^{\#}\right)\right\|}{1-\varsigma L / v}\right)^{2} .
\end{aligned}
$$

Since $\lim _{n \rightarrow \infty} \zeta_{n}=0, \lim _{n \rightarrow \infty}\left\|\phi\left(x_{n+1}\right)-\phi\left(x_{n}\right)\right\|=0$, and $\lim _{n \rightarrow \infty}\left\|\mathbb{B} x_{n}-\mathbb{B} x^{\#}\right\|=0$, we have

$$
\lim _{n \rightarrow \infty}\left\|\phi\left(x_{n}\right)-u_{n}\right\|=0 .
$$

Next, we prove that $\lim \sup _{n \rightarrow \infty}\left\langle\varsigma \varrho\left(x^{*}\right)-\phi\left(x^{*}\right), u_{n}-\phi\left(x^{*}\right)\right\rangle \leq$ 0 , where $x^{*}$ is the unique solution of (7). Let $\left\{u_{n_{i}}\right\}$ be a subsequence of $\left\{u_{n}\right\}$ such that

$$
\begin{aligned}
& \limsup _{n \rightarrow \infty}\left\langle\varsigma \varrho\left(x^{*}\right)-\phi\left(x^{*}\right), u_{n}-\phi\left(x^{*}\right)\right\rangle \\
& \quad=\lim _{i \rightarrow \infty}\left\langle\varsigma \varrho\left(x^{*}\right)-\phi\left(x^{*}\right), u_{n_{i}}-\phi\left(x^{*}\right)\right\rangle \\
& \quad=\lim _{i \rightarrow \infty}\left\langle\varsigma \varrho\left(x^{*}\right)-\phi\left(x^{*}\right), \phi\left(x_{n_{i}}\right)-\phi\left(x^{*}\right)\right\rangle .
\end{aligned}
$$

By the boundedness of $\left\{x_{n_{i}}\right\}$, there exists a subsequence $\left\{x_{n_{i_{j}}}\right\}$ of $\left\{x_{n_{i}}\right\}$ which converges weakly to some point $z \in \mathbb{C}$. Without loss of generality, we may assume that $x_{n_{i}} \rightarrow z$. From the weak continuity of $\phi$, we deduce $\phi\left(x_{n_{i}}\right) \rightarrow \phi(z)$. Next, we prove $z \in \Delta$. We firstly show $z \in E P(\theta, \mathbb{A})$. Noting that $z_{n}=$ $\mathbb{S}_{\tau_{n}}\left(u_{n}-\tau_{n} \mathbb{A} u_{n}\right)$, for any $y \in \mathbb{C}$, we have

$$
\theta\left(z_{n}, y\right)+\frac{1}{\tau_{n}}\left\langle y-z_{n}, z_{n}-\left(u_{n}-\tau_{n} \AA \mathrm{A} u_{n}\right)\right\rangle \geq 0
$$


Since $\theta$ is monotone, we have

$$
\frac{1}{\tau_{n}}\left\langle y-z_{n}, z_{n}-\left(u_{n}-\tau_{n} \mathbb{A} u_{n}\right)\right\rangle \geq \theta\left(y, z_{n}\right), \quad \forall y \in \mathbb{C} .
$$

Hence,

$$
\left\langle y-z_{n_{i}}, \frac{z_{n_{i}}-u_{n_{i}}}{\tau_{n_{i}}}+\mathbb{A} u_{n_{i}}\right\rangle \geq \theta\left(y, z_{n_{i}}\right), \quad \forall y \in \mathbb{C} .
$$

Let $v_{t}=t y+(1-t) z$, for all $t \in(0,1]$ and $y \in C$. We have $v_{t} \in C$. So, from (50) we have

$$
\begin{aligned}
\left\langle v_{t}\right. & \left.-z_{n_{i}}, \mathbb{A} v_{t}\right\rangle \\
\geq & \left\langle v_{t}-z_{n_{i}}, \mathbb{A} v_{t}\right\rangle-\left\langle v_{t}-z_{n_{i}}, \frac{z_{n_{i}}-u_{n_{i}}}{\tau_{n_{i}}}+\mathbb{A} u_{n_{i}}\right\rangle \\
& +\theta\left(v_{t}, z_{n_{i}}\right) \\
= & \left\langle v_{t}-z_{n_{i}}, \mathbb{A} v_{t}-\mathbb{A} z_{n_{i}}\right\rangle+\left\langle v_{t}-z_{n_{i}}, \mathbb{A} z_{n_{i}}-\mathbb{A} u_{n_{i}}\right\rangle \\
& -\left\langle v_{t}-z_{n_{i}}, \frac{z_{n_{i}}-u_{n_{i}}}{\tau_{n_{i}}}\right\rangle+\theta\left(v_{t}, z_{n_{i}}\right) .
\end{aligned}
$$

Note that $\left\|\mathbb{A} z_{n_{i}}-\mathbb{A} u_{n_{i}}\right\| \leq(1 / \eta)\left\|z_{n_{i}}-u_{n_{i}}\right\| \rightarrow 0$. Further, from monotonicity of $\mathbb{A}$, we have $\left\langle v_{t}-z_{n_{i}}, \mathbb{A} v_{t}-\mathbb{A} z_{n_{i}}\right\rangle \geq 0$. Letting $i \rightarrow \infty$ in (51), we have $\left\langle v_{t}-z, \mathbb{A} v_{t}\right\rangle \geq \theta\left(v_{t}, z\right)$. This together with (C1) and (C4) implies that

$$
\begin{aligned}
0 & =\theta\left(v_{t}, v_{t}\right) \leq t \theta\left(v_{t}, y\right)+(1-t) \theta\left(v_{t}, z\right) \\
& \leq t \theta\left(v_{t}, y\right)+(1-t)\left\langle v_{t}-z, \mathbb{A} v_{t}\right\rangle \\
& =t \theta\left(v_{t}, y\right)+(1-t) t\left\langle y-z, \mathbb{A} v_{t}\right\rangle
\end{aligned}
$$

and hence $0 \leq \theta\left(v_{t}, y\right)+(1-t)\left\langle\mathbb{A} v_{t}, y-z\right\rangle$. Letting $t \rightarrow 0$, we have $0 \leq \theta(z, y)+\langle y-z, \mathbb{A} z\rangle$. This implies that $z \in E P(\theta, \mathbb{A})$. Next, we prove $z \in V I(\mathbb{B}, \phi, \mathbb{C})$. Set

$$
R v= \begin{cases}\mathbb{B} v+\mathbb{N}_{\mathbb{C}}(v), & v \in \mathbb{C} \\ \emptyset, & v \notin \mathbb{C}\end{cases}
$$

It is well known that $R$ is maximal $\phi$-monotone. Let $(v, w) \in$ $G(R)$ (the graph of $R$ ). Since $w-\mathbb{B} v \in \mathbb{N}_{\mathbb{C}}(v)$ and $x_{n} \in \mathbb{C}$, we have $\left\langle\phi(v)-\phi\left(x_{n}\right), w-\mathbb{B} v\right\rangle \geq 0$. Noting that $u_{n}=$ $\operatorname{proj}_{\mathbb{C}}\left[\phi\left(x_{n}\right)-\omega_{n} \mathbb{B} x_{n}\right]$, we get

$$
\left\langle\phi(v)-u_{n}, u_{n}-\left[\phi\left(x_{n}\right)-\varpi_{n} \mathbb{B} x_{n}\right]\right\rangle \geq 0 .
$$

It follows that

$$
\left\langle\phi(v)-u_{n}, \frac{u_{n}-\phi\left(x_{n}\right)}{\omega_{n}}+\mathbb{B} x_{n}\right\rangle \geq 0
$$

Then,

$$
\begin{aligned}
\langle\phi(v) & \left.-\phi\left(x_{n_{i}}\right), w\right\rangle \\
\geq & \left\langle\phi(v)-\phi\left(x_{n_{i}}\right), \mathbb{B} v\right\rangle \\
\geq & \left\langle\phi(v)-\phi\left(x_{n_{i}}\right), \mathbb{B} v\right\rangle-\left\langle\phi(v)-u_{n_{i}}, \frac{u_{n_{i}}-\phi\left(x_{n_{i}}\right)}{\omega_{n_{i}}}\right\rangle \\
& -\left\langle\phi(v)-u_{n_{i}}, \mathbb{B} x_{n_{i}}\right\rangle \\
= & \left\langle\phi(v)-\phi\left(x_{n_{i}}\right), \mathbb{B} v-\mathbb{B} x_{n_{i}}\right\rangle+\left\langle\phi(v)-\phi\left(x_{n_{i}}\right), \mathbb{B} x_{n_{i}}\right\rangle \\
& -\left\langle\phi(v)-u_{n_{i}}, \frac{u_{n_{i}}-\phi\left(x_{n_{i}}\right)}{\omega_{n_{i}}}\right\rangle-\left\langle\phi(v)-u_{n_{i}}, \mathbb{B} x_{n_{i}}\right\rangle \\
\geq & -\left\langle\phi(v)-u_{n_{i}}, \frac{u_{n_{i}}-\phi\left(x_{n_{i}}\right)}{\omega_{n_{i}}}\right\rangle-\left\langle\phi\left(x_{n_{i}}\right)-u_{n_{i}}, \mathbb{B} x_{n_{i}}\right\rangle .
\end{aligned}
$$

Since $\left\|\phi\left(x_{n_{i}}\right)-u_{n_{i}}\right\| \rightarrow 0$ and $\phi\left(x_{n_{i}}\right) \rightarrow \phi(z)$, we deduce that $\langle\phi(v)-\phi(z), w\rangle \geq 0$ by taking $i \rightarrow \infty$ in (56). Thus, $z \in R^{-1} 0$ by the maximal $\phi$-monotonicity of $R$. Hence, $z \in V I(\mathbb{B}, \phi, \mathbb{C})$. Therefore, $z \in \Delta$. From (47), we obtain

$$
\begin{aligned}
& \limsup _{n \rightarrow \infty}\left\langle\varsigma \varrho\left(x^{*}\right)-\phi\left(x^{*}\right), u_{n}-\phi\left(x^{*}\right)\right\rangle \\
& \quad=\lim _{i \rightarrow \infty}\left\langle\varsigma \varrho\left(x^{*}\right)-\phi\left(x^{*}\right), \phi\left(x_{n_{i}}\right)-\phi\left(x^{*}\right)\right\rangle \\
& \quad=\left\langle\varsigma \varrho\left(x^{*}\right)-\phi\left(x^{*}\right), \phi(z)-\phi\left(x^{*}\right)\right\rangle \leq 0 .
\end{aligned}
$$

Set $y_{n}=\operatorname{proj}_{\mathbb{C}}\left[\zeta_{n} \varsigma \varrho\left(x_{n}\right)+\left(1-\zeta_{n}\right) z_{n}\right]$, for all $n \geq 0$. Then, we have

$$
\begin{aligned}
\| y_{n}- & \phi\left(x^{*}\right) \|^{2} \\
\leq & \left\langle\zeta_{n} \varsigma \varrho\left(x_{n}\right)+\left(1-\zeta_{n}\right) z_{n}-\phi\left(x^{*}\right), y_{n}-\phi\left(x^{*}\right)\right\rangle \\
\leq & \zeta_{n} \varsigma\left\langle\varrho\left(x_{n}\right)-\varrho\left(x^{*}\right), y_{n}-\phi\left(x^{*}\right)\right\rangle \\
& +\zeta_{n}\left\langle\varsigma \varrho\left(x^{*}\right)-\phi\left(x^{*}\right), y_{n}-\phi\left(x^{*}\right)\right\rangle \\
& +\left(1-\zeta_{n}\right)\left\langle z_{n}-\phi\left(x^{*}\right), y_{n}-\phi\left(x^{*}\right)\right\rangle \\
\leq & \frac{\zeta_{n} L \varsigma}{\nu}\left\|\phi\left(x_{n}\right)-\phi\left(x^{*}\right)\right\|\left\|y_{n}-\phi\left(x^{*}\right)\right\| \\
& +\zeta_{n}\left\langle\varsigma \varrho\left(x^{*}\right)-\phi\left(x^{*}\right), y_{n}-\phi\left(x^{*}\right)\right\rangle \\
& +\left(1-\zeta_{n}\right)\left\|z_{n}-\phi\left(x^{*}\right)\right\|\left\|y_{n}-\phi\left(x^{*}\right)\right\|
\end{aligned}
$$




$$
\begin{aligned}
\leq & \zeta_{n}\left(\frac{\varsigma L}{v}\right)\left\|\phi\left(x_{n}\right)-\phi\left(x^{*}\right)\right\|\left\|y_{n}-\phi\left(x^{*}\right)\right\| \\
& +\zeta_{n}\left\langle\varsigma \varrho\left(x^{*}\right)-\phi\left(x^{*}\right), y_{n}-\phi\left(x^{*}\right)\right\rangle \\
& +\left(1-\zeta_{n}\right)\left\|\phi\left(x_{n}\right)-\phi\left(x^{*}\right)\right\|\left\|y_{n}-\phi\left(x^{*}\right)\right\| \\
= & {\left[1-\left(1-\frac{L \varsigma}{v}\right) \zeta_{n}\right]\left\|\phi\left(x_{n}\right)-\phi\left(x^{*}\right)\right\|\left\|y_{n}-\phi\left(x^{*}\right)\right\| } \\
& +\zeta_{n}\left\langle\varsigma \varrho\left(x^{*}\right)-\phi\left(x^{*}\right), y_{n}-\phi\left(x^{*}\right)\right\rangle \\
= & \frac{1-(1-L \varsigma / v) \zeta_{n}}{2}\left\|\phi\left(x_{n}\right)-\phi\left(x^{*}\right)\right\|^{2}+\frac{1}{2}\left\|y_{n}-\phi\left(x^{*}\right)\right\|^{2} \\
& +\zeta_{n}\left\langle\varsigma \varrho\left(x^{*}\right)-\phi\left(x^{*}\right), y_{n}-\phi\left(x^{\#}\right)\right\rangle .
\end{aligned}
$$

It follows that

$$
\begin{aligned}
\left\|y_{n}-\phi\left(x^{*}\right)\right\|^{2} \leq & {\left[1-\left(1-\frac{L \varsigma}{\nu}\right) \zeta_{n}\right]\left\|\phi\left(x_{n}\right)-\phi\left(x^{*}\right)\right\|^{2} } \\
& +2 \zeta_{n}\left\langle\varsigma \varrho\left(x^{*}\right)-\phi\left(x^{*}\right), y_{n}-\phi\left(x^{*}\right)\right\rangle .
\end{aligned}
$$

Therefore,

$$
\begin{aligned}
& \left\|\phi\left(x_{n+1}\right)-\phi\left(x^{*}\right)\right\|^{2} \\
& \leq \eta_{n}\left\|\phi\left(x_{n}\right)-\phi\left(x^{*}\right)\right\|^{2}+\left(1-\eta_{n}\right)\left\|y_{n}-\phi\left(x^{*}\right)\right\|^{2} \\
& \leq \eta_{n}\left\|\phi\left(x_{n}\right)-\phi\left(x^{*}\right)\right\|^{2} \\
& +\left(1-\eta_{n}\right)\left[1-\left(1-\frac{\varsigma L}{v}\right) \zeta_{n}\right]\left\|\phi\left(x_{n}\right)-\phi\left(x^{*}\right)\right\|^{2} \\
& +2\left(1-\eta_{n}\right) \zeta_{n}\left\langle\varsigma \varrho\left(x^{*}\right)-\phi\left(x^{*}\right), y_{n}-\phi\left(x^{*}\right)\right\rangle \\
& =\left[1-\left(1-\frac{\varsigma L}{\nu}\right)\left(1-\eta_{n}\right) \zeta_{n}\right]\left\|\phi\left(x_{n}\right)-\phi\left(x^{*}\right)\right\|^{2} \\
& +2\left(1-\eta_{n}\right) \zeta_{n}\left\langle\varsigma \varrho\left(x^{*}\right)-\phi\left(x^{*}\right), y_{n}-\phi\left(x^{*}\right)\right\rangle \\
& =\left[1-\left(1-\frac{\varsigma L}{\nu}\right)\left(1-\eta_{n}\right) \zeta_{n}\right]\left\|\phi\left(x_{n}\right)-\phi\left(x^{*}\right)\right\|^{2} \\
& +\left(1-\frac{\varsigma L}{\nu}\right)\left(1-\eta_{n}\right) \zeta_{n} \\
& \times\left(\frac{2}{1-\varsigma L / \nu}\left\langle\varsigma \varrho\left(x^{*}\right)-\phi\left(x^{*}\right), y_{n}-\phi\left(x^{*}\right)\right\rangle\right) \\
& =\left(1-v_{n}\right)\left\|\phi\left(x_{n}\right)-\phi\left(x^{*}\right)\right\|^{2}+\varsigma_{n} v_{n} \text {, }
\end{aligned}
$$

where $\nu_{n}=(1-\varsigma L / \nu)\left(1-\eta_{n}\right) \zeta_{n}$ and $\varsigma_{n}=(2 /(1-\varsigma L / \nu))$ $\left\langle\varsigma \varrho\left(x^{*}\right)-\phi\left(x^{*}\right), y_{n}-\phi\left(x^{*}\right)\right\rangle$. It is easily seen that $\sum_{n} \nu_{n}=\infty$. Since

$$
\begin{aligned}
\left\|y_{n}-u_{n}\right\| & \leq\left\|y_{n}-z_{n}\right\|+\left\|z_{n}-u_{n}\right\| \\
& \leq \zeta_{n}\left\|\varsigma \phi\left(x_{n}\right)-z_{n}\right\|+\left\|z_{n}-u_{n}\right\| \\
& \longrightarrow 0
\end{aligned}
$$

and by $\lim \sup _{n \rightarrow \infty}\left\langle\varsigma \varrho\left(x^{*}\right)-\phi\left(x^{*}\right), u_{n}-\phi\left(x^{*}\right)\right\rangle \leq 0$, we get $\lim \sup _{n \rightarrow \infty} \varsigma_{n} \leq 0$. We can therefore apply Lemma 3 to conclude that $\phi\left(x_{n}\right) \rightarrow \phi\left(x^{*}\right)$ and $x_{n} \rightarrow x^{*}$. This completes the proof.

\section{Conflict of Interests}

The authors declare that there is no conflict of interests regarding the publication of this paper.

\section{Acknowledgments}

The authors are extremely grateful to the anonymous referees for their useful comments and suggestions. This study was supported by research funds from Dong-A University.

\section{References}

[1] E. Blum and W. Oettli, "From optimization and variational inequalities to equilibrium problems," The Mathematics Student, vol. 63, no. 1-4, pp. 123-145, 1994.

[2] P. L. Combettes and S. A. Hirstoaga, "Equilibrium programming in Hilbert spaces," Journal of Nonlinear and Convex Analysis, vol. 6, no. 1, pp. 117-136, 2005.

[3] Y. Yao, Y. J. Cho, and Y.-C. Liou, "Algorithms of common solutions for variational inclusions, mixed equilibrium problems and fixed point problems," European Journal of Operational Research, vol. 212, no. 2, pp. 242-250, 2011.

[4] G. Stampacchia, "Formes bilinéaires coercitives sur les ensembles convexes," Comptes Rendus de l'Académie des Sciences, vol. 258, pp. 4413-4416, 1964.

[5] G. M. Korpelevič, "An extragradient method for finding saddle points and for other problems," Ekkonomika i Matematicheskie Metody, vol. 12, no. 4, pp. 747-756, 1976.

[6] R. Glowinski, Numerical Methods for Nonlinear Variational Problems, Springer, New York, NY, USA, 1984.

[7] A. N. Iusem, "An iterative algorithm for the variational inequality problem," Computational and Applied Mathematics, vol. 13, no. 2, pp. 103-114, 1994.

[8] M. A. Noor, "Some developments in general variational inequalities," Applied Mathematics and Computation, vol. 152, no. 1, pp. 199-277, 2004.

[9] F. Facchinei and J.-S. Pang, Finite-Dimensional Variational Inequalities and ComplementarityProblems, Volume 1, Springer Series in Operations Research, Springer, New York, NY, USA, 2003.

[10] F. Facchinei and J.-S. Pang, Finite-Dimensional Variational Inequalities and Complementarity Problems, Volume 2, Springer Series in Operations Research, Springer, New York, NY, USA, 2003.

[11] H. K. Xu and T. H. Kim, "Convergence of hybrid steepestdescent methods for variational inequalities," Journal of Optimization Theory and Applications, vol. 119, no. 1, pp. 185-201, 2003.

[12] J. C. Yao, "Variational inequalities with generalized monotone operators," Mathematics of Operations Research, vol. 19, no. 3, pp. 691-705, 1994.

[13] L.-C. Zeng and J.-C. Yao, "Strong convergence theorem by an extragradient method for fixed point problems and variational 
inequality problems," Taiwanese Journal of Mathematics, vol. 10, no. 5, pp. 1293-1303, 2006.

[14] L.-C. Ceng, S. Al-Homidan, Q. H. Ansari, and J.-C. Yao, "An iterative scheme for equilibrium problems and fixed point problems of strict pseudo-contraction mappings," Journal of Computational and Applied Mathematics, vol. 223, no. 2, pp. 967-974, 2009.

[15] M. A. Noor, "New approximation schemes for general variational inequalities," Journal of Mathematical Analysis and Applications, vol. 251, no. 1, pp. 217-229, 2000.

[16] R. Glowinski and P. Le Tallec, Augmented Lagrangian and Operator-Splitting Methods in Nonlinear Mechanics, vol. 9 of SIAM Studies in Applied Mathematics, SIAM, Philadelphia, Pa, USA, 1989.

[17] S. Haubruge, V. H. Nguyen, and J. J. Strodiot, "Convergence analysis and applications of the Glowinski-Le Tallec splitting method for finding a zero of the sum of two maximal monotone operators," Journal of Optimization Theory and Applications, vol. 97, no. 3, pp. 645-673, 1998.

[18] Y. Censor and T. Elfving, "A multiprojection algorithm using Bregman projections in a product space," Numerical Algorithms, vol. 8, no. 2-4, pp. 221-239, 1994.

[19] C. Byrne, "Iterative oblique projection onto convex sets and the split feasibility problem," Inverse Problems, vol. 18, no. 2, pp. 441453, 2002.

[20] H.-K. Xu, "Iterative methods for the split feasibility problem in infinite-dimensional Hilbert spaces," Inverse Problems, vol. 26, no. 10, Article ID 105018, 17 pages, 2010.

[21] T. Suzuki, "Strong convergence theorems for infinite families of nonexpansive mappings in general Banach spaces," Fixed Point Theory and Applications, no. 1, pp. 103-123, 2005.

[22] H.-K. Xu, "Iterative algorithms for nonlinear operators," Journal of the London Mathematical Society, vol. 66, no. 1, pp. 240-256, 2002. 


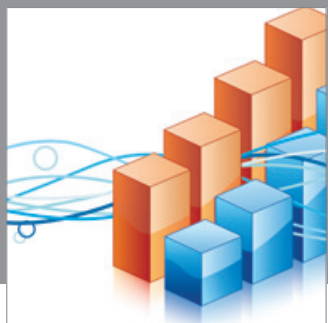

Advances in

Operations Research

mansans

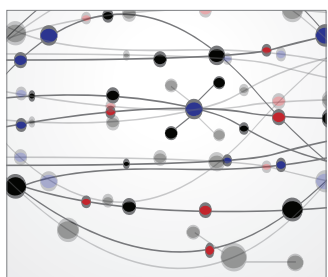

The Scientific World Journal
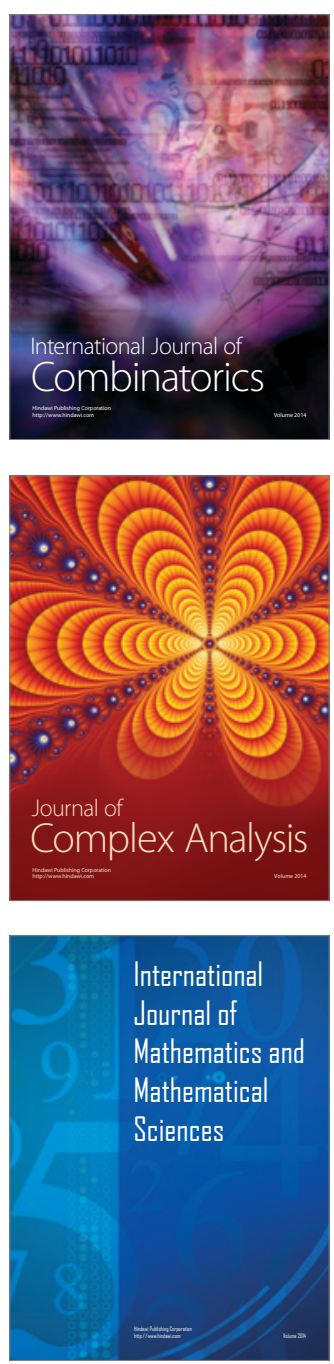
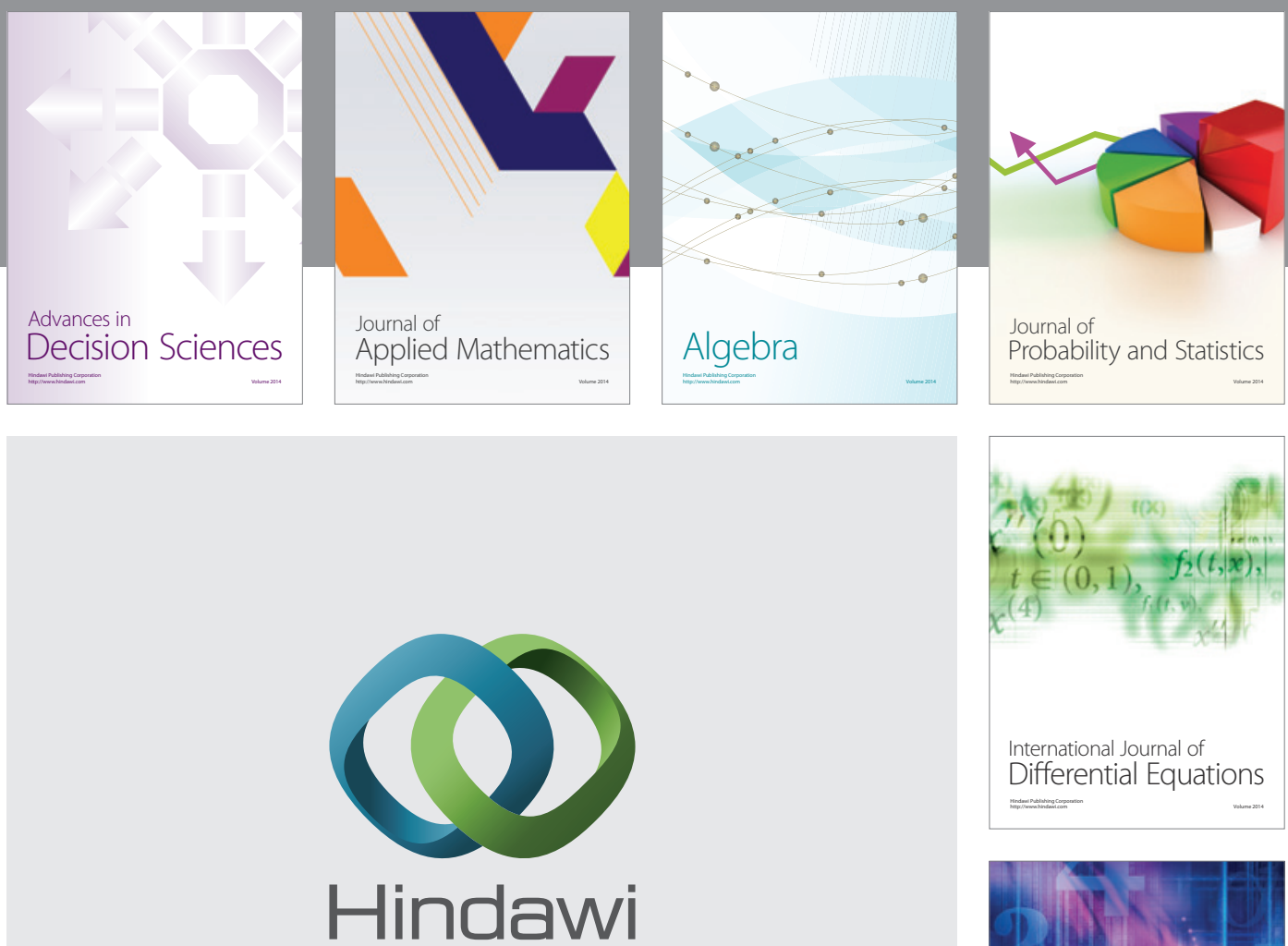

Submit your manuscripts at http://www.hindawi.com
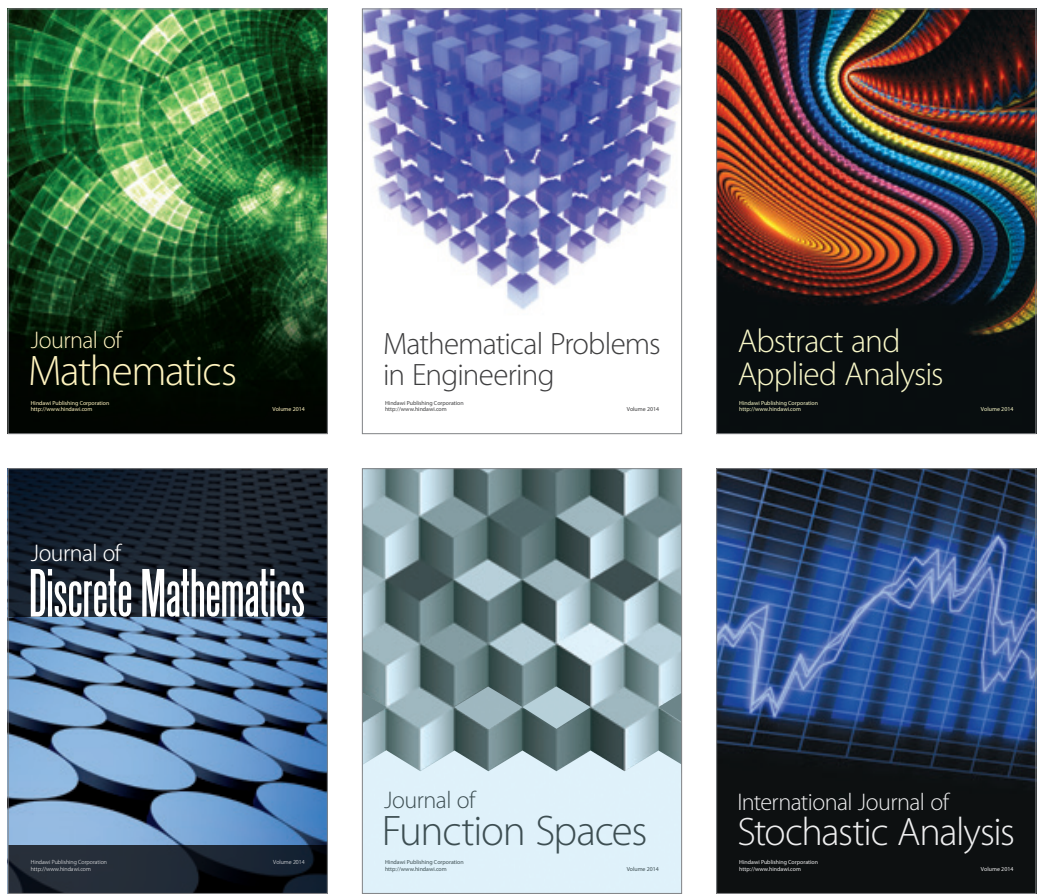

Journal of

Function Spaces

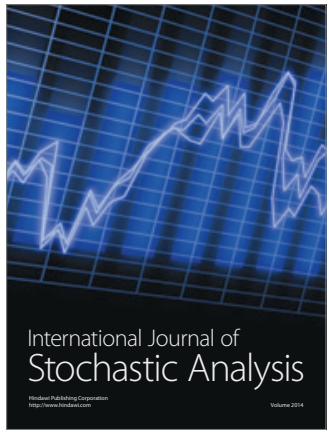

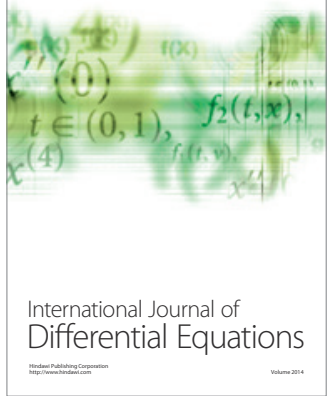
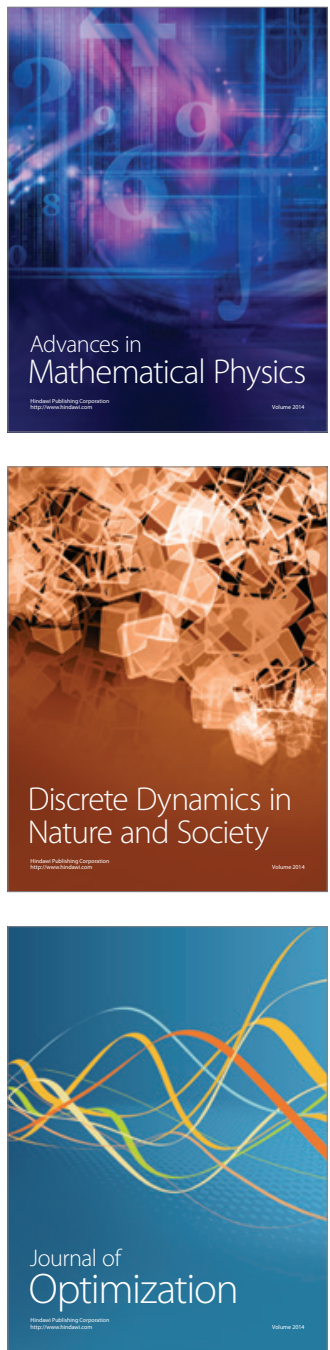